\title{
Mid-infrared waveform measurement by rapid mechanical scanning
}

\author{
Philip Jacob $^{1}$, Alexander Weigel ${ }^{1,2}$, David Gröters ${ }^{2}$, Theresa Buberl ${ }^{1}$, Michael Trubetskov ${ }^{1}$, Marinus \\ Huber $^{1,2}$, Joachim Heberle ${ }^{3}$, Ioachim Pupeza ${ }^{1,2}$ \\ 1. Max-Planck-Institut für Quantenoptik, Hans-Kopfermann-Str. 1, Garching 85748, Germany \\ 2. Fakultät für Physik, Ludwig-Maximilians-Universität München, Am Coulombwall 1, Garching 85748, Germany \\ 3. Department of Physics, Experimental Molecular Biophysics, Freie Universität Berlin, Arnimallee 14, Berlin 14195, Germany
}

Many applications of ultrafast lasers, such as pump-probe spectroscopy [1], 2D femtosecond spectroscopy [2] and field resolving electro-optic sampling (EOS) [3] demand the scan of one or more optical delays between laser pulses. Conventionally, this is realized by moving a retroreflector with a mechanical stage, typically restricting the scan rate to the $\mathrm{Hz}$ regime at best. Laser intensity noise, however, has major contributions from sub-kHz components that directly influence the scan results. Performing full optical scans at multi-kHz rates can eliminate signal distortion by such low-frequency noise components. In addition, repeating full optical scans on sub-ms time scales allows to follow chemical reactions in real time [4]. Several approaches have been pursued in the past to achieve picosecond-delay optical scans at $\mathrm{kHz}$ rates, including for example electronically controlled synchronization of two laser oscillators [5] and acousto-optic delay generation [6]. Many of the proposed techniques are either costly and complex or place restrictions on the wavelength and bandwidth of the laser pulses.

In this work we amplify the motion of a resonantly oscillating piezo with a sonotrode to perform forward/backward scans of a mirror over a 1.5-ps range at an effective rate of $38 \mathrm{kHz}$. The sonotrode scanner provides a simple and cost-effective way to upgrade a conventional femtosecond experiment to ultrafast scan speeds without wavelength or spectral bandwidth limitations. Its application has been previously shown for timeresolved FTIR spectroscopy with a non-coherent light source [4]. Here we demonstrate for the first time the use of a sonotrode in a complex non-linear optical experiment with femtosecond pulses: The mapping of the electric field of a few-cycle mid-infrared (mid-IR) pulse generated by intra-pulse difference frequency generation of compressed pulses from an Yb:YAG oscillator $(28 \mathrm{MHz}, 60 \mathrm{~W}, 17 \mathrm{fs})$ by EOS [3]. We use the interferometric modulation of an auxiliary continuous-wave beam to precisely track the resonant motion of the moving mirror in real time (Fig. 1a). By applying a predictor-corrector algorithm we were able to perform consistent calibration of the delay-time axis over several seconds of integration time, with a time-precision reaching 111 attoseconds for a single scan, and 14 attoseconds for 100 scans. Accurate knowledge of the delay axis is necessary for harnessing the advantages of fast scanning by averaging multiple scans or tracking signal evolution from chemical reactions in a scan series. The inset in Fig. 1b shows how oscillations which are overpowered by noise in a single scan become visible upon averaging. The baseline noise scales with the square root of the number of traces averaged (Fig. 1c), so that with $1 \mathrm{~s}$ acquisition time, a dynamic range of $>10^{8}$ is reached.

In conclusion, we have shown EOS of few-cycle mid-IR pulses with a simple mechanical multi-kHz scanner, while maintaining attosecond precision and reproducibility for the delay axis.

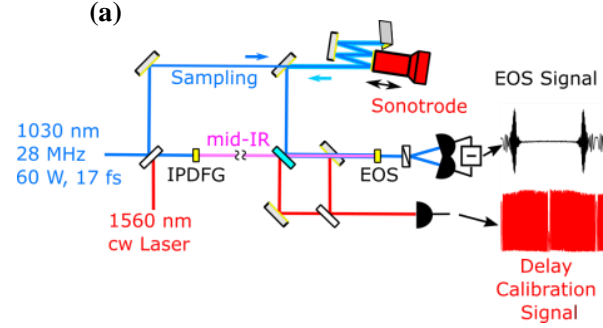

(b)

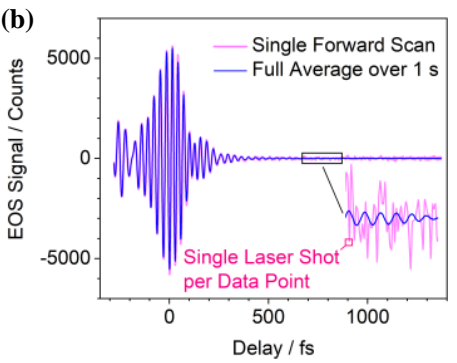

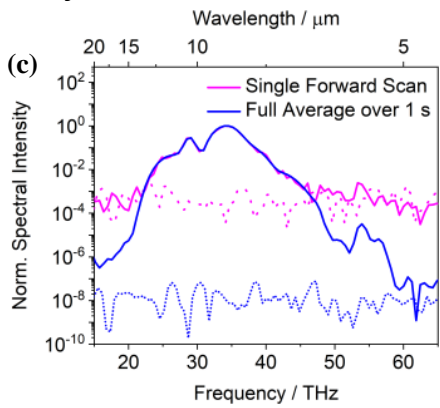

Fig. 1. (a) Schematic diagram of the experimental setup. (b) Comparison of a single EOS scan mapping the electric field of the mid-infrared pulse with the mean of all traces acquired over 1s (i.e., 38000 scans). (c) Mid-IR intensity spectra obtained by Fourier transformation of the traces in (b) (Dashed lines represent baseline noise).

\section{References}

[1] A. Zewail, "Femtochemistry: Atomic-Scale Dynamics of the Chemical Bond Using Ultrafast Lasers (Nobel Lecture)", Angew. Chem. Int. Ed. 39, 2586-2631 (2000).

[2] D.M. Jonas, "Two-dimensional femtosecond spectroscopy", Annu. Rev. Phys. Chem. 54, 425-463 (2003)

[3] I. Pupeza et al., "Field-resolved infrared spectroscopy of biological systems," Nature 577, 52-59 (2020).

[4] B. Süss, F. Ringleb, and J. Heberle,."New ultrarapid-scanning interferometer for FT-IR spectroscopy with microsecondtime-resolution," Rev. Sci. Instrum. 87, 063113 (2016)

[5] S. Kray, F. Spöler, T. Hellerer, and H. Kurz, "Electronically controlled coherent linear optical sampling for optical coherence tomography," Opt. Express 18, 9976-9990 (2010)

[6] O. Schubert, M. Eisele, V. Crozatier, N. Forget, D. Kaplan, and R. Huber, "Rapid-scan acousto-optical delay line with 34 kHz scan rate and 15 as precision," Opt. Lett. 38, 2907-2910 (2013) 